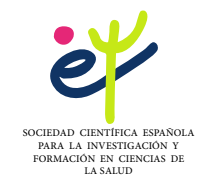

European Journal of Education and Psychology

2021, Vol. 14, No 1 (Págs. 1-14)

Eur. j. educ. psychol. e-ISSN 1989-2209 https://revistas.uautonoma.cl/index.php/ejep doi: $10.32457 /$ ejep.v14i1.1400

\title{
Flexibilidad en el afrontamiento del estrés y fortalezas personales en estudiantes universitarios
}

\section{Coping flexibility and personal strengths in university students}

\author{
María del Mar Ferradás Canedo* (D), Carlos Freire Rodríguez \\ y Pablo González Rico \\ Universidade da Coruña. Facultad de Ciencias de la Educación, Campus de Elviña, La Coruña, España
}

\begin{abstract}
Resumen
Las altas y heterogéneas demandas que ha de afrontar el estudiantado universitario en su día a día han acrecentado en los últimos años el interés de la investigación por el estudio de la flexibilidad en el afrontamiento como recurso eficaz para la consecución de un funcionamiento psicológico adaptativo. Desde este planteamiento, el presente trabajo analizó la relación entre la utilización flexible de diferentes estrategias de afrontamiento del estrés y las fortalezas personales, erigidas en competencias psicológicas esenciales para la consecución de una vida plena, satisfactoria y feliz. Participaron 401 estudiantes de la Universidade da Coruña (España). Mediante un análisis de perfiles latentes (LPA) se identificaron dos perfiles diferenciados de afrontamiento: un perfil de baja flexibilidad en el afrontamiento (preferencia por estrategias de autocrítica y retirada social) y un perfil de alta flexibilidad en el afrontamiento (preferencia por estrategias de resolución de problemas, reestructuración cognitiva, expresión emocional y apoyo social). Este último grupo evidenció niveles significativamente más elevados de fortalezas personales que el grupo de afrontamiento improductivo. De conformidad con estos hallazgos, las intervenciones psicoeducativas dirigidas a la adquisición y utilización estratégica de diferentes recursos aproximativos de afrontamiento podrían constituir una vía eficaz para favorecer el desarrollo de las fortalezas personales en el estudiantado universitario.

Palabras clave: estrategias de afrontamiento, afrontamiento flexible, estrés académico, fortalezas personales, estudiantes universitarios.
\end{abstract}

\footnotetext{
* Para correspondencia: Correo: mar.ferradasc@udc.es. Facultad de Ciencias de la Educación, Campus de Elviña s/n, 15071 A Coruña (España).
} 


\begin{abstract}
The high and heterogeneous demands that university students have to face in their day-to-day lives have increased in recent years the research interest for the study of coping flexibility as an effective resource for achieving adaptive psychological functioning. From this approach, the present work analyzed the relationship between coping flexibility and personal strengths, established as essential psychological skills for achieving a full, satisfying and happy life. 401 students from the University of a Coruna (Spain) participated. Through a latent profile analysis (LPA), two differentiated coping profiles were identified: a profile of low coping flexibility (preference for self-criticism strategies and social withdrawal) and a profile of high coping flexibility (preference for problem-solving strategies, cognitive restructuring, emotional expression and social support). The last profile showed significantly higher levels of personal strengths than the unproductive coping profile. In accordance with these findings, psychoeducational interventions aimed at the acquisition and strategic use of different approach coping resources could constitute an effective way to favor the development of personal strengths in university students.
\end{abstract}

Keywords: coping strategies, coping flexibility, academic stress, personal strengths, university students

\title{
INTRODUCCIÓN
}

Los niveles de estrés están alcanzando cotas sin precedentes en la población estudiantil universitaria (Liu, Stevens, Wong, Yasui y Chen, 2019). A las exigencias propias del trabajo académico, entre las que se encontrarían la sobrecarga de tareas, el escaso tiempo para acometerlas o la presión por obtener un buen rendimiento (Webber, Skodda, Muth, Angerer y Loerbroks, 2019), se añadirían otras de tipo interpersonal, laboral y financieras (Acharya, Jin y Collins, 2018; Bulo y Sánchez, 2014). Las dificultades para gestionar de forma equilibrada este amplio volumen de demandas conlleva, en no pocos casos, importantes repercusiones para la salud física y mental, así como para el rendimiento académico (American College Health Association, 2018; Mortier et al., 2018; Shankar y Park, 2016).

Estas perniciosas consecuencias han acrecentado la atención en los últimos años hacia los mecanismos de afrontamiento desplegados por el estudiantado universitario para dar respuesta al estrés. En efecto, "el modo en que las personas afrontan el estrés puede reducir o incrementar las consecuencias de los eventos vitales adversos, no solo en términos de malestar emocional y funcionamiento a corto plazo, sino también a largo plazo, en el desarrollo de problemas de salud y patologías físicas y mentales" (Skinner, Edge, Altman y Sherwood, 2003, p. 216). 
El afrontamiento tiene su marco conceptual de referencia en la teoría transaccional del estrés (Folkman y Moskowitz, 2004). Esta teoría postula que, ante una determinada demanda, externa o interna, el individuo realiza un doble proceso de valoración cognitiva. En primer término, se analiza en qué medida el suceso puede constituir un reto o, en cambio, una amenaza para el bienestar personal. En este último caso, el individuo sopesa la disponibilidad de recursos y estrategias para hacer frente de forma efectiva a la situación. Este planteamiento, ampliamente aceptado en la investigación subraya, así pues, que, en aras de desarrollar todo su potencial de aprendizaje, es preciso que los estudiantes dispongan de estrategias adaptativas que les permitan hacer frente de forma efectiva a los diferentes retos y obstáculos que inevitablemente se van a encontrar en su día a día (Skinner y Saxton, 2019).

El amplio y heterogéneo elenco de estrategias cognitivas y conductuales que el estudiantado puede poner en marcha para hacer frente al estrés ha sido habitualmente categorizado en dos grandes tipologías: aproximativas y evasivas (Zimmer-Gembeck y Skinner, 2016). Las primeras abarcarían todos aquellos esfuerzos dirigidos a la gestión directa de la situación o de las emociones subyacentes a ella, entre las que se encontrarían la planificación, la búsqueda de una solución al problema, la reevaluación positiva del mismo o la búsqueda de ayuda con fines instrumentales y/o de apoyo emocional. Por el contrario, las estrategias evasivas suponen todo intento por evitar cualquier situación o circunstancia potencialmente amenazante, como por ejemplo, ignorar la demanda, negarla, escapar de ella o distraer la mente.

Si bien la adopción de estrategias de afrontamiento de corte aproximativo se asocia a un mayor ajuste en esta etapa académica (e.g., Sawhney, Kunen y Gupta, 2020), la extensa diversidad de exigencias que el estudiantado debe asumir en su día a día ha motivado un creciente interés investigador por la capacidad para flexibilizar las respuestas de afrontamiento en función de las características concretas de la situación (para un metaanálisis, véase Cheng, Lau y Chan, 2014). En esta línea, los estudios sobre flexibilidad en el afrontamiento asumen la compatibilidad entre diferentes estrategias (Eisenbarth, 2012), bajo la premisa de que algunas personas disponen de un amplio abanico de mecanismos de afrontamiento, mostrando una alta capacidad para simultanearlas (Nielsen y Knardahl, 2014), mientras otras tienden a aferrarse sistemáticamente a la misma estrategia, o a un número reducido de ellas, independientemente del tipo de demanda a afrontar (Cheng y Cheung, 2005).

En el ámbito universitario, varios trabajos han documentado la funcionalidad de los perfiles flexibles en sus respuestas de afrontamiento, mostrando, en comparación con los perfiles menos flexibles, una menor vulnerabilidad psicopatológica (Dodek et al., 2020; Hasselle, Schwartz, Berlin y Howell, 2019), así como niveles más elevados de bienestar psicológico (Freire, Ferradás, Núñez y Valle, 2018). Esta incipiente línea de investigaciones abre la puerta al estudio de los recursos psicológicos personales que contribuyen a que los 
estudiantes más flexibles en su afrontamiento del estrés logren estados de salud mental más adaptativos. En este sentido, Freire et al., (2020) han evidenciado recientemente que la flexibilidad en el afrontamiento se relaciona positivamente con las expectativas de autoeficacia del estudiantado universitario.

De conformidad con este planteamiento proactivo, el presente estudio pretende analizar la relación entre la flexibilidad en el afrontamiento del estrés en la etapa universitaria y las fortalezas personales. El constructo fortalezas personales constituye uno de los núcleos neurálgicos de la Psicología Positiva, centrada en la identificación de los recursos psicológicos que se relacionan con una vida plena, satisfactoria y feliz (Seligman, 2011). Con este propósito, Peterson y Seligman (2004) evidenciaron empíricamente la existencia de veinticuatro fortalezas del carácter en el ser humano. Estas fortalezas representarían de forma universal y ubicua los rasgos (i.e., acciones, pensamientos y sentimientos) positivos y moralmente valiosos que se vinculan con las seis grandes virtudes del ser humano (sabiduría y conocimiento, espiritualidad y trascendencia, valor, humanidad, justicia y templanza).

El auge suscitado por el estudio científico de estas fortalezas personales ha llevado a evidenciar, entre otros aspectos, su relación con la resiliencia (Martínez-Martí y Ruch, 2017), la satisfacción vital (Ruch, Gander, Platt y Hofmann, 2018) o el bienestar subjetivo y psicológico (Hausler et al., 2017). En lo tocante al estrés en la etapa universitaria, la escasa investigación precedente apunta a la existencia de una vinculación entre las fortalezas personales y las estrategias de afrontamiento de índole aproximativa (Freire, Ferradás y González-Rico, 2019; Gustems-Carnicer y Calderón, 2016). Estos trabajos, sin embargo han analizado la relación entre fortalezas y estrategias sin tomar en consideración la perspectiva de la flexibilidad en el afrontamiento. En consecuencia, la principal aportación de este trabajo radica en analizar la posible existencia de diferencias significativas en cuanto a las fortalezas personales de los estudiantes en función de su perfil de afrontamiento. Apoyándonos en los trabajos revisados, se espera que aquellos estudiantes con un perfil más flexible en el afrontamiento del estrés muestren niveles de fortalezas personales significativamente más altos que los estudiantes con menor flexibilidad.

\section{MÉTODO}

\section{Participantes}

El estudio contó con la participación de 401 estudiantes universitarios (326 mujeres y 75 varones; $M_{\text {edad }}=21.82 ; D T=3.04$ ) pertenecientes a la Universidade da Coruña (España), seleccionados mediante un muestreo por conveniencia. Los participantes cursaban sus estudios en los grados de Educación Infantil $(n=228 ; 56.9 \%)$ y Educación Primaria $(n=$ 
$173 ; 43.1 \%)$. Se obtuvo representatividad muestral de los cuatro cursos: primero $(n=67$; $16.7 \%)$, segundo $(n=89 ; 22.2 \%)$, tercero $(n=152 ; 37.9 \%)$ y cuarto $(n=93 ; 23.2 \%)$.

\section{Instrumentos}

Estrategias de afrontamiento delestrés. Se utilizó el Inventario de Estrategias deAfrontamiento (Tobin, Holroyd, Reynolds y Kigal, 1989), en su adaptación española (Cano, Rodríguez y García, 2007). El instrumento mide cuatro estrategias aproximativas de afrontamiento (resolución de problemas, expresión emocional, apoyo social y reestructuración cognitiva) y cuatro estrategias evasivas (autocrítica, pensamiento desiderativo, evitación de problemas y retirada social). Se utilizó una escala de respuesta tipo Likert $(0=$ En absoluto $-4=$ Totalmente). La consistencia interna de los ocho factores osciló entre $\alpha=.73$ (evitación de problemas) y $\alpha=.89$ (autocrítica).

Fortalezas personales. El instrumento utilizado fue el Test breve de Fortalezas Personales (Park, Peterson y Seligman, 2004), en su adaptación española (Saldaña et al., 2014). Consta de 24 ítems $(\alpha=.78)$, uno en representación de cada fortaleza personal. Las respuestas de los participantes se registraron en una escala Likert $(1=$ Nunca $-5=$ Siempre $)$.

\section{Procedimiento}

El protocolo de investigación se realizó en consonancia con los estándares éticos recogidos en la Declaración de Helsinki, con consentimiento informado por escrito de los participantes, voluntariedad de la participación y confidencialidad y anonimato de las respuestas. La recogida de datos se realizó en una única sesión sin límite de tiempo, en los propios centros y aulas donde los participantes cursaban sus estudios.

\section{Análisis de datos}

Los perfiles de flexibilidad en el afrontamiento se determinaron mediante un análisis de perfiles latentes — LPA - (Lanza, Flaherty y Collins, 2003) con el programa estadístico MPlus 7.11 (Muthén y Muthén, 1998-2012). Para ello, se estimó, de entre un conjunto finito de modelos, cuál se ajusta mejor a los datos. El modelo óptimo se seleccionó de acuerdo con los siguientes parámetros: criterio de información de Akaike (AIC), criterio de información bayesiana de Schwarz (BIC), BIC ajustado por el tamaño de la muestra (SSABIC), prueba formal de la razón de máxima verosimilitud ajustada de Lo, Mendell y Rubin (2001) -LMRT - y prueba paramétrica de la razón de verosimilitud de reemplazamiento (PBLRT). Asimismo, se tomó como criterio de exclusión la existencia de clases espurias ( $n$ $\leq 5 \%$ de la muestra). Una vez seleccionado el modelo óptimo, se procedió a determinar su 
precisión clasificatoria tomando como referencia el cálculo de las probabilidades a posteriori y el estadístico de entropía.

Finalmente, las diferencias en fortalezas personales entre los perfiles de afrontamiento identificados se estimaron mediante un ANOVA. La magnitud del efecto de las diferencias entre grupos se determinó mediante el estadístico eta cuadrado parcial: efecto nulo $\left(\eta_{\mathrm{p}}{ }^{2}<\right.$ $.01)$; pequeño $\left(.01>\eta_{\mathrm{p}}{ }^{2}<.058\right)$; mediano $\left(.059>\eta_{\mathrm{p}}{ }^{2}<.137\right)$; y grande $\left(\eta_{\mathrm{p}}^{2} \geq .138\right)$. Estos análisis se ejecutaron con el programa estadístico SPSS 26.0 (IBM Corp, 2019).

\section{RESULTADOS}

\section{Estadísticos descriptivos y análisis de correlaciones}

En la Tabla 1 se muestran los estadísticos descriptivos y las correlaciones (Pearson) de las variables del estudio. Los valores de la asimetría y la curtosis de las variables fueron indicativos de una distribución normal. Las fortalezas personales evidenciaron correlaciones positivas significativas con las cuatro estrategias aproximativas $(p<.001$, en todos los casos). En cuanto a las estrategias evasivas, la retirada social correlacionó negativa y significativamente $(r=-.16, p<.05)$ con las fortalezas personales.

\section{Tabla 1}

Medias, desviaciones típicas y correlaciones entre las estrategias de afrontamiento del estrés y las fortalezas personales

\begin{tabular}{lccccccccc}
\hline & 1 & 2 & 3 & 4 & 5 & 6 & 7 & 8 & 9 \\
\hline 1. REP & - & & & & & & & & \\
2. AUC & -.02 & - & & & & & & & \\
3. EEM & $.20^{* *}$ & -.01 & - & & & & & & \\
4. PSD & $-.11^{*}$ & $31^{* *}$ & $.14^{* *}$ & - & & & & & \\
5. APS & $.27^{* *}$ & -.08 & $.51^{* *}$ & .09 & - & & & & \\
6. REC & $.42^{* *}$ & .03 & $.26^{*}$ & $-.13^{*}$ & $.40^{* *}$ & - & & & \\
7. EVP & .08 & .07 & -.01 & .01 & .04 & $.44^{* *}$ & - & & \\
8. RES & $-.18^{*}$ & $.41^{* *}$ & $-.34^{* *}$ & $.31^{* *}$ & $-.31^{* *}$ & -.07 & $.21^{*}$ & - & \\
9. Fortalezas & $.34^{* *}$ & .01 & $.27^{* *}$ & .02 & $.29^{* *}$ & $.28^{* *}$ & .03 & $-.16^{*}$ & - \\
M & 2.80 & 1.72 & 2.23 & 2.82 & 2.74 & 2.22 & 1.42 & 1.30 & 2.90 \\
DT & 0.82 & 1.14 & 1.07 & 1.03 & 0.98 & 0.93 & 0.87 & 1.00 & 0.37 \\
Asimetría & -0.67 & 0.07 & -0.11 & -0.85 & -0.72 & -0.27 & 0.31 & 0.52 & -0.31 \\
Curtosis & 0.33 & -1.02 & -0.95 & -0.22 & -0.20 & -0.51 & -0.60 & -0.60 & 0.30 \\
\hline
\end{tabular}

Nota: $R E P=$ Resolución de Problemas; $A U C=$ Autocritica $; E E M=$ Expresión Emocional; $P S D=$ Pensamiento Desiderativo; APS = Apoyo Social $; R E C=$ Reestructuración Cognitiva $; E V P=$ Evitación de Problemas; $R E S=$ Retirada Social . ${ }^{*} p<.05,{ }^{* *} p<.001$ 


\section{Identificación y caracterización de los perfiles de afrontamiento}

Se analizó el ajuste de varios modelos de perfiles latentes (modelos de dos a cuatro clases; véase Tabla 2). En el ajuste de modelos se asumió que las varianzas podían diferir entre los indicadores dentro de cada grupo, especificándose como restricción que fuesen iguales entre los grupos. Asimismo, se impuso como restricción la independencia entre los indicadores, tanto dentro de cada grupo como entre grupos.

Analizando los diferentes parámetros evaluados en los tres modelos, se observó que el modelo de dos clases era el único que mostraba valores LMRT y PBLRT estadísticamente significativos $(p<.05)$, condición indicativa de que los modelos de más clases (i.e., modelos de tres y cuatro clases) no ajustan mejor que el modelo precedente. Por tanto, pese a que otros estadísticos, como el AIC, BIC y SSA-BIC o la entropía, muestran mejores valores de ajuste en los modelos de tres y cuatro clases que en el de dos clases, se considera que estos valores son meramente descriptivos, de manera que la decisión final debe tomarse con base en los indicadores LMRT y PBLRT (Muthén y Muthén, 1998-2012). Por ello, se optó por seleccionar el modelo de dos clases como el más apropiado.

\section{Tabla 2}

Estadísticos para la identificación del ajuste de modelos de clases latentes y precisión clasificatoria.

\begin{tabular}{lrrr}
\hline & \multicolumn{1}{c}{ Dos clases } & \multicolumn{1}{c}{ Tres clases } & Cuatro clases \\
\hline AIC & 8759.425 & 8635.224 & 8529.786 \\
BIC & 8859.274 & 8771.018 & 8701.526 \\
SSA-BIC & 8779.947 & 8663.134 & 8565.084 \\
Entropía & .703 & .779 & .761 \\
Número de grupos con $n \leq 5 \%$ & 0 & 0 & 0 \\
LMRT & $256.647^{*}$ & 139.614 & 121.191 \\
PBLRT & $261.404^{* *}$ & $142.202^{* *}$ & $123.438^{* *}$ \\
\hline
\end{tabular}

Nota: $A I C=$ Criterio de Información de Akaike; BIC = Criterio de información bayesiana de Schwarz; SSA$B I C=B I C$ ajustado al tamaño muestral; $L M R T=$ prueba formal de la razón de máxima verosimilitud ajustada de Lo, Mendell y Rubin; PBLRT = prueba paramétrica de la razón de verosimilitud de reemplazamiento; ${ }^{*} p<.01 ;{ }^{* *} p<.001$

La Tabla 3 informa de la precisión clasificatoria del modelo de dos clases. Los promedios asociados a los grupos a los que se asignaron los participantes se presentan en la diagonal principal de la tabla, en negrita. El primer grupo evidenció un coeficiente de clasificación del $87 \%$ y el segundo grupo, del $94 \%$. Asimismo, el valor del estadístico de entropía de este modelo (.70; véase Tabla 2) es indicativo de una precisión razonable. Así pues, en conjunto, estos datos indican que el modelo de dos clases presenta una adecuada precisión clasificatoria. 


\section{Tabla 3}

Caracterización de los perfiles latentes y precisión clasificatoria de los sujetos en cada perfil

\begin{tabular}{lccc} 
& \multicolumn{4}{c}{ Perfiles latentes } \\
& 1 & \multicolumn{3}{c}{2} & $n(\%)$ \\
1. PBF & & & \\
2. PAF & $\mathbf{. 8 7 1}$ & .129 & $160(39.9)$ \\
& .061 & .939 & $241(60.1)$ \\
\hline
\end{tabular}

Nota: PBF: perfil de baja flexibilidad en el afrontamiento; PAF: perfil de alta flexibilidad en el afrontamiento. En negrita se muestran los coeficientes asociados a los grupos a los que se asignaron los participantes.

En la Tabla 4 se muestran las puntuaciones medias (directas y estandarizadas) de los sujetos asignados a cada una de las dos clases. El primer grupo ( $n=160 ; 39.9 \%)$, lo conforman estudiantes con una alta utilización de las estrategias de autocrítica y retirada social, así como un bajo empleo del resto de estrategias consideradas. De acuerdo con esta caracterización, hemos denominado a este grupo como perfil de baja flexibilidad en el afrontamiento (PBF). El segundo grupo $(n=241 ; 60.1 \%)$ se caracteriza por una elevada utilización de las cuatro estrategias aproximativas consideradas (resolución de problemas, expresión emocional, apoyo social y reestructuración cognitiva), combinado con un bajo empleo de las estrategias de autocrítica y retirada social, así como un muy moderado uso del pensamiento desiderativo y la evitación de problemas. Este grupo de estudiantes ha recibido la consideración de perfil de alta flexibilidad en el afrontamiento (PAF). La representación gráfica de estos perfiles se muestra en la Figura 1.

Tabla 4

Descripción de los perfiles latentes (medias, desviaciones típicas e intervalos de confianza)

\begin{tabular}{lrrrr} 
& \multicolumn{4}{c}{ Intervalos de confianza } \\
\cline { 2 - 5 } & $M$ & $D T$ & Inferior 5\% & Superior 5\% \\
\hline PBF $(n=160)$ & & & & \\
Resolución de problemas & $2.45(-0.39)$ & 0.12 & 2.25 & 2.65 \\
Autocrítica & $1.86(1.04)$ & 0.16 & 1.59 & 2.13 \\
Expresión emocional & $1.38(-0.82)$ & 0.14 & 1.14 & 1.62 \\
Pensamiento desiderativo & $2.78(-0.01)$ & 0.15 & 2.54 & 3.02 \\
Apoyo social & $1.91(-0.85)$ & 0.17 & 1.64 & 2.19 \\
Reestructuración cognitiva & $1.73(-0.53)$ & 0.14 & 1.50 & 1.96 \\
Evitación de problemas & $1.35(-0.04)$ & 0.09 & 1.20 & 1.50 \\
Retirada social & $1.83(0.56)$ & 0.14 & 1.60 & 2.06 \\
PAF $(n=241)$ & & & & \\
Resolución de problemas & $3.00(0.26)$ & 0.05 & 2.91 & 3.08 \\
\hline
\end{tabular}




\begin{tabular}{lrrrr} 
& & & \multicolumn{2}{c}{ Intervalos de confianza } \\
\hline & $M$ & $D T$ & Inferior 5\% & Superior 5\% \\
\hline Autocrítica & $1.64(-0.07)$ & 0.10 & 1.48 & 1.80 \\
Expresión emocional & $2.76(0.54)$ & 0.16 & 2.49 & 3.03 \\
Pensamiento desiderativo & $2.82(0.01)$ & 0.10 & 2.67 & 2.98 \\
Apoyo social & $3.25(0.56)$ & 0.08 & 3.11 & 3.38 \\
Reestructuración cognitiva & $2.53(0.35)$ & 0.07 & 2.41 & 2.64 \\
Evitación de problemas & $1.45(0.02)$ & 0.07 & 1.34 & 1.56 \\
Retirada social & $0.97(-0.37)$ & 0.08 & 0.83 & 1.11 \\
\hline
\end{tabular}

Nota: PBF: perfil de baja flexibilidad en el afrontamiento; PAF: perfil de alta flexibilidad en el afrontamiento. Las puntaciones medias normalizadas (z) figuran entre paréntesis.

\section{Figura 1.}

Representación gráfica de los perfiles de flexibilidad en el afrontamiento (puntuaciones estandarizadas)

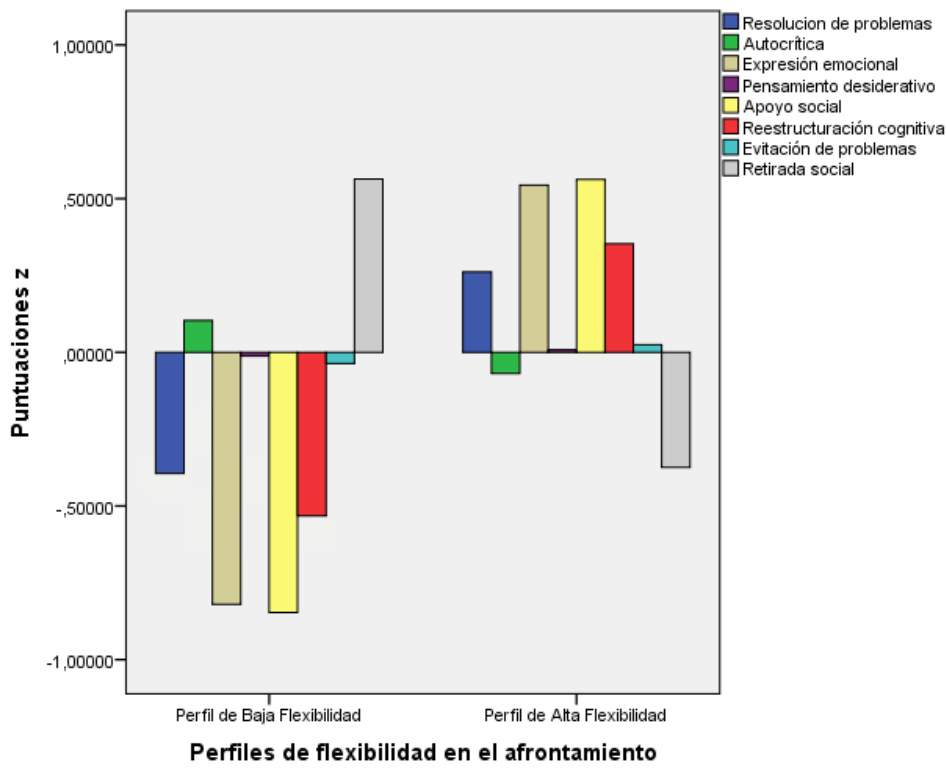

\section{Relación entre los perfiles de afrontamiento y las fortalezas personales}

El PAF obtuvo una puntuación media $M=2.99(D T=0.33)$ en fortalezas personales. Por su parte, el PBF evidenció una puntuación inferior $(M=2.76 ; D T=0.37)$ en esta variable. Los resultados del ANOVA de un factor evidenciaron diferencias estadísticamente 
significativas entre ambos perfiles de flexibilidad en el afrontamiento en cuanto a sus fortalezas personales $\left(F_{400}=43.125, p<.001, \eta_{\mathrm{p}}^{2}=.098\right)$, siendo mediano el tamańo del efecto.

\section{DISCUSIÓN}

La elevada vulnerabilidad al estrés existente en la etapa universitaria ha focalizado el interés investigador hacia los procesos de afrontamiento. La investigación reciente constata la necesidad de que el estudiantado disponga de un amplio abanico de estrategias para acomodar su respuesta a la demanda específica de la situación como medida eficaz para lograr estados psicológicos adaptativos (Dodek et al., 2020; Freire et al., 2018; Hasselle et al., 2019). Sin embargo existe todavía poco conocimiento sobre los recursos psicológicos personales que explicarían la relación entre flexibilidad en el afrontamiento del estrés y salud mental. Desde esta consideración, la principal contribución de este estudio ha sido analizar la relación entre perfiles de afrontamiento en el alumnado universitario y fortalezas personales, estas últimas erigidas en uno de los pilares esenciales para la consecución de una vida placentera, comprometida y con significado (Park et al., 2004).

Los resultados obtenidos nos han permitido identificar la existencia de dos perfiles bien diferenciados de estudiantes en virtud de su flexibilidad en el afrontamiento de las demandas universitarias cotidianas. Un primer grupo de estudiantes se caracterizaría por recurrir de forma prioritaria a estrategias evasivas como la autocrítica y la retirada social. Se trataría, así pues, de estudiantes con un perfil poco flexible y altamente disfuncional para hacer frente a los estresores, en la medida en que tienden a responder a estos reprochándose sistemáticamente su inhabilidad para darles una solución efectiva y aislándose socialmente, sin compartir sus preocupaciones y dificultades. Por el contrario, la tendencia de estos estudiantes a utilizar estrategias dirigidas al control, ya sea primario o secundario, de la situación sería muy baja. Estas características, como así indican otros estudios (Cheng et al., 2014; Nielsen y Knardahl, 2014), hacen del alumnado encuadrado en este perfil un claro candidato a experimentar perjuicios en su salud mental.

El otro grupo identificado, parece evidenciar un patrón de afrontamiento ciertamente más adaptativo que el anterior, por cuanto muestra una elevada utilización de las cuatro estrategias aproximativas analizadas (resolución de problemas, expresión emocional, apoyo social y reestructuración cognitiva), conjugada con una baja o muy modesta recurrencia a las estrategias evasivas. Estos estudiantes, por consiguiente, exhibirían una elevada flexibilidad en sus respuestas de afrontamiento, combinando estrategias dirigidas al control directo de los estresores (resolución de problemas y apoyo social) con otras encaminadas a regular con eficacia las emociones difíciles que pueden derivarse de estas situaciones (expresión emocional, reestructuración cognitiva). Desde esta consideración, podría asumirse que el 
estudiantado que encarna este perfil goza de mayores recursos para responder adaptativamente a las diferentes demandas de su día a día, desplegando estrategias de control primario ante estresores controlables y estrategias de control secundario ante estresores incontrolables (Zimmer-Gembeck y Skinner, 2016).

Los resultados del presente estudio parecen corroborar la mayor funcionalidad de este segundo perfil, por cuanto, de conformidad con lo hipotetizado, su puntuación en fortalezas personales fue significativamente más elevada que la del perfil de baja flexibilidad en el afrontamiento. Este dato, así pues, es consistente con los estudios que, desde un enfoque centrado en la variable, encontraron una relación positiva entre estrategias aproximativas de afrontamiento y fortalezas personales (Freire et al., 2019; Gustems-Carnicer y Calderón, 2016). Asimismo, la relación entre flexibilidad en el afrontamiento y fortalezas personales evidenciada en este trabajo se suma a la incipiente línea de trabajos que vinculan la disponibilidad de recursos psicológicos personales con la capacidad del estudiantado universitario para conjugar de forma adaptativa distintas estrategias frente al estrés (e.g., Freire et al., 2020).

Una importante implicación derivada de nuestros hallazgos anida en la conveniencia de diseñar intervenciones específicas encaminadas a incrementar la capacidad del alumnado universitario para responder de forma efectiva a la amplia diversidad de exigencias que, inevitablemente, se le presentan en su día a día. En este sentido, es preciso que estas intervenciones se focalicen, no solo en ampliar el repertorio de estrategias de afrontamiento, sino también las habilidades metacognitivas del estudiantado para seleccionar la estrategia o estrategias más apropiadas en cada situación (Cheng y Cheung, 2005).

Las aportaciones de este estudio, no obstante, deben analizarse a la luz de algunas limitaciones, principalmente vinculadas al diseño de investigación y características de la muestra. Respecto al primero, el carácter transversal del estudio no permite establecer relaciones de causalidad entre los perfiles identificados y las fortalezas personales. En cuanto a la muestra, su escaso tamaño, así como el procedimiento de reclutamiento realizado restringen la generalización de los resultados obtenidos al conjunto de la población universitaria. Se precisan, por tanto, nuevos trabajos con diseños metodológicos más rigurosos para confirmar la existencia de los perfiles de afrontamiento identificados en este estudio, así como su relación con las fortalezas personales.

\section{REFERENCIAS}

Acharya, L., Lan, J. \& Collins, W. (2018). College life is stressful today-Emerging stressors and depressive symptoms in college students. Journal of American College Health, 66(7), 655-664. https://doi.org/10.1080/07448481.2018.1451869 
American College Health Association (2018). National College Health Assessment II: Reference Group Executive Summary Fall 2018. Hanover, MD: American College Health Association. https://www.acha.org/documents/ncha/NCHA-II_Fall_2018_ Reference_Group_Executive_Summary.pdf

Bulo, J.G. \& Sánchez, M.G. (2014). Sources of stress among college students. CVCITC Research Journal, 1(1), 16-25.

Cano, F.J., Rodríguez, L. \& García, J. (2007). Adaptación española del Inventario de Estrategias de Afrontamiento. Actas Españolas de Psiquiatría, 35(1), 29-39. https:// idus.us.es/handle/11441/56854

Cheng, C. \& Cheung, M.W.L. (2005). Cognitive processes underlying coping flexibility: differentiation and integration. Journal of Personality, 73, 859-886. https://doi. org/10.1111/j.1467-6494.2005.00331.x

Cheng, C., Lau, H.-P.B. \& Chan, M.P.S. (2014). Coping flexibility and psychological adjustment to stressful life changes: A meta-analytic review. Psychological Bulletin, 140, 1582-1607. https://doi.org/10.1037/a0037913

Dodek, P. M., Culjak, A., Cheung, E. O., Hubinette, M. M., Holmes, C., Schrewe, B., ... Crowell, P. (2020). Active coping in medical students is associated with less burnout and higher resilience. American Journal of Respiratory and Critical Care Medicine, 201:A4300. https://doi.org/10.1164/ajrccm-conference.2019.199.1_ MeetingAbstracts.A4300

Eisenbarth, C. (2012). Coping profiles and psychological distress: A cluster analysis. North American Journal of Psychology, 14, 485-496.

Folkman, S. \& Moskowitz, J. T. (2004). Coping: Pitfalls and promise. Annual Review of Psychology, 55, 745-774. https://doi.org/10.1146/annurev.psych.55.090902.141456

Freire, C., Ferradás, M.M. \& González-Rico, P. (2019). Capacidad predictiva de las estrategias de afrontamiento del estrés sobre las fortalezas personales en estudiantes universitarios. En VVAA (Eds.), Innovación docente e investigación en Ciencias Sociales, Económicas y Jurídicas (pp. 875-884). Madrid: Dykinson.

Freire, C., Ferradás, M.M., Núñez, J.C. \& Valle, A. (2018). Coping flexibility and eudaimonic well-being in university students. Scandinavian Journal of Psychology, 59, 433-442. https://doi.org/10.1111/sjop.12458

Freire, C., Ferradás, M.M., Regueiro, B., Rodríguez, S., Valle, A. \& Núñez, J.C. (2020) Coping strategies and self-efficacy in university students: A person-centered approach. Frontiers in Psychology, 11:841. https://doi.org/10.3389/fpsyg.2020.00841

Gustems-Carnicer, J. \& Calderón, C. (2016). Virtues and character strengths related to approach coping strategies of college students. Social Psychology of Education, 19(1), 77-95. https://doi.org/10.1007/s11218-015-9305-y 
Hasselle, A.J., Schwartz, L.E., Berlin, K.S. \& Howell, K.H. (2019). A latent profile analysis of coping responses to individuals' most traumatic event: associations with adaptive and maladaptive mental health outcomes. Anxiety Stress and Coping 32, 626-640. https://doi.org/10.1080/10615806.2019.1638733

Hausler, M., Strecker, C., Huber, A., Brenner, M., Höge, T. \& Höfer, S. (2017). Distinguishing relational aspects of character strengths with subjective and psychological well-being. Frontiers in Psychology, 8:1159. https://doi.org/10.3389/ fpsyg.2017.01159

IBM Corp (2019). SPSS STATISTICS for Windows, Version 26.0. Armonk, NY: IBM Corp.

Lanza, S.T., Flaherty, B.P. \& Collins, L.M. (2003). Latent class and latent transition analysis. En Schinka, J. A., y Velicer, W. F. (Eds.), Handbook of Psychology: Research Methods in Psychology (pp. 663-685), Hobobken, NJ: Wiley. https://doi. org/10.1002/0471264385.wei0226

Liu, C.H., Stevens, C., Wong, S.H.M., Yasui, M. \& Chen, J.A. (2019). The prevalence and predictors of mental health diagnoses and suicide among U.S. college students: Implications for addressing disparities in service use. Depress Anxiety, 36: 8-17. https://doi.org/10.1002/da.22830

Lo, Y., Mendell, N.R. \& Rubin, D.B. (2001). Testing the number of components in a normal mixture. Biometrika, 88, 767-778. https://doi.org/10.1093/biomet/88.3.767

Martínez-Martí, M.L. \& Ruch, W. (2017). Character strengths predict resilience over and above positive affect, self-efficacy, optimism, social support, self-esteem, and life satisfaction. The Journal of Positive Psychology, 12, 110-119. https://doi.org/10.1080 /17439760.2016.1163403

Mortier, P., Auerbach, R.P., Alonso, J., Bantjes, J., Benjet, C., Cuipers, P., ... Kessler, R.C. (2018). Suicidal thoughts and behaviors among firstyear college students: Results from the WMH-ICS project. Journal of the American Academy of Child \& Adolescent Psychiatry, 57(4), 263-273. https://doi.org/10.1016/j.jaac.2018.01.018

Muthén, L.K. \& Muthén, B.O. (1998-2012). Mplus User's Guide, Gth ed. Los Angeles, CA: Muthén \& Muthén.

Nielsen, M.B. \& Knardahl, S. (2014). Coping strategies: A prospective study of patterns, stability, and relationships with psychological distress. Scandinavian Journal of Psychology, 55(2), 142-150. https://doi.org/10.1111/sjop.12103

Park, N., Peterson, C. \& Seligman, M.E.P. (2004). Strengths of character and well-being. Journal of Social and Clinical Psychology, 23(5), 603-619. https://doi.org/10.1521/ jscp.23.5.603.50748

Peterson, C. \& Seligman, M.E.P. (2004). Character strengths and virtues: A handbook and classification. Washington, DC: American Psychological Association. 
Ruch, W., Gander, F., Platt, T. \& Hofmann, J. (2018). Team roles: Their relationships to character strengths and job satisfaction. The Journal of Positive Psychology, 13(2), 190-199. https://doi.org/10.1080/17439760.2016.1257051

Saldaña, O., Escartín, J., Martín-Peña, J., Jiménez, Y., Ceja, L., Varela, A., ...Rodríguez, A. (2014, septiembre). Fortalezas personales relacionadas con el rendimiento académico y profesional en el campo de la psicología social y la psicología social aplicada. Trabajo presentado en el I Congreso Internacional de Educación Emocional, Barcelona, España. Disponible en http://diposit.ub.edu/dspace/bitstream/2445/58743/1/ C150_162.pdf

Sawhney, M., Kunen, S. \& Gupta, A. (2020). Depressive symptoms and coping strategies among Indian university students. Psychological Reports, 123(2), 266-280. https:// doi.org/10.1177/0033294118820511

Seligman, M.E.P. (2011). La vida que florece. Barcelona: Ediciones B.

Shankar, N.L. \& Park, C.L. (2016). Effects of stress on students' physical and mental health and academic success. International Journal of School \& Educational Psychology, 4(1), 5-9. https://doi.org/10.1080/21683603.2016.1130532

Skinner, E.A., Edge, K., Altman, J. \& Sherwood, H. (2003) Searching for the structure of coping: A review and critique of category systems for classifying ways of coping. Psychological Bulletin, 129, 216-269. https://doi.org/10.1037/0033-2909.129.2.216.

Skinner, E.A. \& Saxton, E.A. (2019). The development of academic coping in children and youth: A comprehensive review and critique. Developmental Psychology, 53. https:// doi.org/10.1016/j.dr.2019.100870

Tobin, D.L., Holroyd, K.A., Reynolds, R.V. \& Kigal, J.K. (1989). The hierarchical factor structure of the Coping Strategies Inventory. Cognitive Therapy and Research, 13(4), 343-361. https://doi.org/10.1007/BF01173478

Webber, J., Skodda, S., Muth, T., Angerer, P. \& Loerbroks, A. (2019). Stressors and resources related to academic studies and improvements suggested by medical students: a qualitative study. BMC Medical Education, 19:312. https://doi.org/10.1186/ s12909-019-1747-z

Zimmer-Gembeck, M.J. \& Skinner, E.A. (2016). The development of coping: Implications for psychopathology and resilience. En D. Cicchetti (Ed.), Developmental psychology: Risk, resilience, and intervention (pp. 485-545). New York, NY: John Wiley \& Sons. https://doi.org/10.1002/9781119125556.devpsy410

Recibido: 15 de noviembre de 2020

Aceptado: 08 de enero de 2021 